\title{
Perioperative management of warfarin and antiplatelet therapy
}

\section{ABSTRACT}

Perioperative management of patients on warfarin or antiplatelet therapy involves assessing and balancing individual risks for thromboembolism and bleeding. Discontinuing anticoagulant and antiplatelet therapy is usually necessary for major surgery but increases the risk of thrombotic events. Bridge therapy, the temporary perioperative substitution of low-molecular-weight heparin or unfractionated heparin in place of warfarin, is an effective means of reducing the risk of thromboembolism but may increase the risk of bleeding. The timing of warfarin withdrawal and timing of the preoperative and postoperative components of bridge therapy are critical to balancing these risks. Perioperative management of antiplatelet therapy requires special care in patients with coronary stents; the timing of surgery relative to stent placement dictates management in these patients.

\section{KEY POINTS}

Determining when and how to use bridge anticoagulation therapy depends on the patient's risk for thromboembolism, which is in turn based on the indication for warfarin-ie, a mechanical heart valve, atrial fibrillation, or prior venous thromboembolism.

Factor patient preference into whether and how to use bridge therapy: many patients are more concerned about stroke risk than bleeding risk, regardless of the relative frequency of these events.

Anticoagulation with warfarin often does not need to be interrupted for patients undergoing minor surgery, such as some ophthalmic, dental, dermatologic, and gastrointestinal procedures.

Premature discontinuation of antiplatelet therapy in surgical patients with recent coronary stent placement significantly raises the risk of catastrophic perioperative stent thrombosis.

See end of article for author disclosures. doi:10.3949/ccjm.76.s4.07

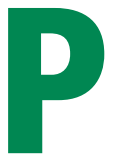

erioperative management of surgical patients who require temporary discontinuation of vitamin $\mathrm{K}$ antagonists (warfarin) or antiplatelet drugs is complicated. The risk of a thrombotic event during interruption of anticoagulant or antiplatelet therapy must be weighed against the risk of bleeding when such therapy is used in close proximity to a surgical procedure. This balancing of risks is guided by the patient's individual risk for thromboembolism or bleeding and underlying conditions such as the presence of a mechanical heart valve or a coronary stent.

High-profile adverse events have made anticoagulant and antiplatelet management one of the most highly litigated aspects of perioperative medicine. Moreover, there is a paucity of randomized clinical trial data and definitive guidelines to address the perioperative needs of patients on antithrombotic therapy. Treatment protocols vary depending on many underlying factors, such as the presence of mechanical heart valves, comorbidities, stent type and location, patient age and medical history, and type of surgical procedure. While recent attention has focused on genetic variations that result in higher or lower sensitivity to warfarin in some patients, routine genetic testing for warfarin sensitivity is controversial and not part of widespread practice at this time.

The first portion of this article explores key issues and principles in the perioperative management of surgical patients on warfarin therapy, and the second portion does the same for surgical patients on antiplatelet therapy.

\section{ACCP RECOMMENDATIONS FOR PERIOPERATIVE ANTICOAGULANT MANAGEMENT}

In 2008 the American College of Chest Physicians (ACCP) published the latest update of its consensus guidelines for the perioperative management of patients receiving antithrombotic therapy. ${ }^{1}$ The guidelines' recommendations for anticoagulant management are based on stratification of patients into risk categories (Table 1) according to their underlying indication for longterm anticoagulation - ie, presence of a mechanical heart valve, history of atrial fibrillation, or history of venous thromboembolism (VTE).

Patients with mechanical valves who are at high risk for perioperative thromboembolism include those with any mechanical mitral valve, an older valve, or a history of stroke or transient ischemic attack (TIA). Patients 
TABLE 1

ACCP's suggested risk stratification for perioperative thromboembolism*

\begin{tabular}{|c|c|c|c|}
\hline Risk category & Mechanical heart valve & Atrial fibrillation & Venous thromboembolism \\
\hline \multicolumn{4}{|l|}{ High } \\
\hline (>10\%/yr risk of ATE & Any mechanical mitral valve & $\mathrm{CHADS}_{2}$ score of 5 or 6 & Recent $(<3$ mo) VTE \\
\hline \multirow[t]{2}{*}{ or $>10 \% /$ mo risk of VTE) } & Older aortic valve & Recent $(<3 \mathrm{mo})$ stroke or TIA & Severe thrombophilia \\
\hline & Recent $(<6 \mathrm{mo})$ stroke or TIA & Rheumatic valvular heart disease & \\
\hline \multicolumn{4}{|l|}{ Moderate } \\
\hline \multirow[t]{2}{*}{$\begin{array}{l}\text { ( } 4 \%-10 \% / y \text { r risk of ATE } \\
\text { or } 4 \%-10 \% / \text { mo risk of VTE) }\end{array}$} & $\begin{array}{l}\text { Bileaflet aortic valve and } \\
\text { one of the following: }\end{array}$ & $\mathrm{CHADS}_{2}$ score of 3 or 4 & $\begin{array}{l}\text { VTE within past 3-12 mo } \\
\text { Recurrent VTE }\end{array}$ \\
\hline & $\begin{array}{l}\text { atrial fibrillation, prior stroke/TIA, } \\
\text { hypertension, diabetes, heart failure, } \\
\text { age }>75 \mathrm{yr}\end{array}$ & & $\begin{array}{l}\text { Nonsevere thrombophilic conditions } \\
\text { Active cancer }\end{array}$ \\
\hline \multicolumn{4}{|l|}{ Low } \\
\hline $\begin{array}{l}(<4 \% / y r \text { risk of } \mathrm{ATE} \\
\text { or }<2 \% / \mathrm{mo} \text { risk of } \mathrm{VTE})\end{array}$ & $\begin{array}{l}\text { Bileaflet aortic valve without } \\
\text { atrial fibrillation and no other } \\
\text { risk factors for stroke }\end{array}$ & $\begin{array}{l}\text { CHADS }_{2} \text { score of } 0-2 \\
\text { (and no prior stroke or TIA) }\end{array}$ & $\begin{array}{l}\text { Single VTE within past } 12 \mathrm{mo} \\
\text { and no other risk factors }\end{array}$ \\
\hline
\end{tabular}

*Reproduced, with permission of American College of Chest Physicians, from Chest (Douketis et al. The perioperative management of antithrombotic therapy. Chest 2008;

133(suppl):299S-339S), copyright (C) 2008.

$\mathrm{ACCP}=$ American College of Chest Physicians; $\mathrm{ATE}=$ arterial thromboembolism; VTE $=$ venous thromboembolism; $\mathrm{TIA}=$ transient ischemic attack

with atrial fibrillation who are at high risk include those with a recent stroke or TIA, rheumatic valvular heart disease, or a $\mathrm{CHADS}_{2}$ score of 5 or 6 . (The $\mathrm{CHADS}_{2}$ scoring system assigns one point each for a history of congestive heart failure, hypertension, age greater than 75 years, or diabetes, and two points for history of stroke or TIA.) Patients with a history of VTE within the prior 3 months are also considered high risk.

Bridging anticoagulation (bridge therapy) - ie, the temporary use of intravenous unfractionated heparin (IV UFH) or low-molecular-weight heparin (LMWH) prior to surgery-is central to the ACCP's recommendations for perioperative management in patients on long-term anticoagulant therapy. Key ACCP recommendations ${ }^{1}$ for these patients, according to their risk for thromboembolism (Table 1), are as follows:

- High risk-bridging anticoagulation with therapeutic-dose subcutaneous LMWH or IV UFH

- Moderate risk-bridging anticoagulation with therapeutic-dose subcutaneous LMWH, therapeuticdose IV UFH, or low-dose subcutaneous LMWH

- Low risk-bridging anticoagulation with lowdose subcutaneous LMWH or no bridging.

\section{ASSESSING RISKS:} DETERMINING WHETHER TO BRIDGE

Considerations in bridge therapy include balancing the risk of thromboembolism against the risk of bleeding, either of which can lead to catastrophic results. ${ }^{2}$ Though the objective of bridge therapy is to avoid bleeding com- plications associated with invasive procedures, the bridge protocol itself can introduce additional serious complications. Figure 1 presents an algorithm for identifying patient and surgical risk factors for patients on anticoagulation therapy who are undergoing elective surgery.

\section{Patient-specific risk factors}

Patient risk factors include the indication for anticoagulation, as detailed above, as well as other individual risks for thromboembolism, as discussed in the article by Michota on preventing VTE on page S45 of this supplement.

If anticoagulation is indicated because the patient has a mechanical heart valve, the valve type and position must be considered because these factors affect thromboembolic risk, as reflected in Table 1. For instance, the risk of thromboembolism is greater when the valve is in the mitral position than in the aortic position, and is also greater with an older caged-ball valve than with a newer-generation bileaflet valve. ${ }^{3}$

In patients receiving anticoagulation because of atrial fibrillation, annual stroke risk can be estimated using the validated $\mathrm{CHADS}_{2}$ scoring system, as presented in Table 2. ${ }^{4}$ Generally, patients with atrial fibrillation who have a $\mathrm{CHADS}_{2}$ score of 3 or higher should receive bridge therapy, while those with a $\mathrm{CHADS}_{2}$ score of 2 or lower probably should not.

\section{Procedure-related risk factors}

Surgical risks factors include the type of surgery and its associated risks of bleeding and thromboembolism, as 


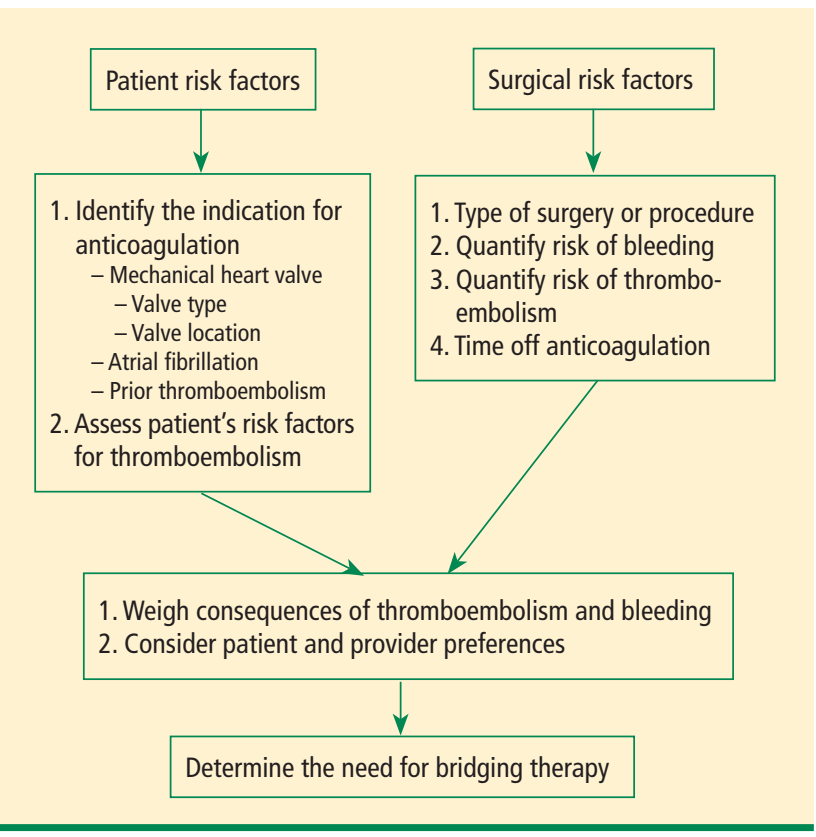

FIGURE 1. Assessment tool for identifying patient-specific and surgical risk factors for patients on anticoagulation therapy who are undergoing elective surgery.

well as the expected time that anticoagulation will be interrupted. Estimating thromboembolic risk is complicated, however, and reliable results are generally not achieved with simplistic calculations or formulas. Such calculations tend not to appropriately account for the hypercoagulable state induced by surgery itself, as the risk of VTE is estimated to be 100 times greater during the perioperative period than in the nonoperative setting, owing to increased levels of plasminogen activator inhibitor-1. Moreover, multiple studies have demonstrated increases in coagulation factors that suggest that a "rebound hypercoagulability" may occur shortly after discontinuation of oral anticoagulant therapy. ${ }^{5-8}$

\section{Net benefit vs risk in trials of bridge therapy}

Several prospective studies of bridge therapy have been conducted in more than 2,700 surgical patients with mechanical heart valves, atrial fibrillation, or prior VTE. ${ }^{9-14}$ Warfarin was discontinued in these patients and replaced with LMWH as bridge therapy. As shown in Table 3, the rate of thromboembolism at follow-up ( 2 weeks to 90 days) in these studies averaged approximately $1 \%$, while the risk of major bleeding was approximately $3.5 \%{ }^{9-14}$

In an analysis of data from observational studies, Kearon and Hirsh estimated the relative risk reduction for thromboembolism with bridge therapy to be $66 \%$ to $80 \%$, depending on the indication for anticoagulation. ${ }^{8}$ Thus, if a patient's risk of developing thromboembolism is $1.5 \%$, bridge therapy reduces the risk to $0.5 \%$ or less.
TABLE 2

Annual stroke risk in patients with atrial fibrillation, according to $\mathrm{CHADS}_{2}$ score

$\begin{array}{cc}\text { CHADS }_{2} \text { score }^{*} & \text { Adjusted stroke rate } \\ 0 & 1.9(1.2-3.0) \\ 1 & 2.8(2.0-3.8) \\ 2 & 4.0(3.1-5.1) \\ 3 & 5.9(4.6-7.3) \\ 4 & 8.5(6.3-11.1) \\ 5 & 12.5(8.2-17.5) \\ 6 & 18.2(10.5-27.4)\end{array}$

* Assessment of the following comorbidities: congestive heart failure, hypertension, age $\geq 75$, and diabetes (1 point each), plus history of stroke or transient ischemic attack (2 points).

${ }^{\dagger}$ Expected rate of stroke per 100 patient-years

Reproduced, with permission, from Snow et al. ${ }^{4}$

Weigh relative consequences of an event with the patient Determining whether and how to initiate bridge therapy ultimately depends on the consequences of an event. Recurrent VTE is fatal in 5\% to $10 \%$ of cases, ${ }^{15}$ and arterial thromboembolism is fatal in $20 \%$ of cases and causes permanent disability in at least $50 \%$ of cases. ${ }^{16}$ While $9 \%$ to $13 \%$ of major bleeding events are fatal, bleeding rarely causes permanent disability. ${ }^{17}$ Thus, whereas a patient who bleeds can be resuscitated, a patient who develops a thromboembolism may be permanently disabled. These considerations should be shared with the patient, and patient preference should factor into the management strategy. Though the risk of bleeding with anticoagulation may be much higher than the risk of stroke without it, many patients will be more concerned about stroke risk.

\section{CHOICE OF AGENT FOR BRIDGE THERAPY}

\section{LMWH appears to offer cost advantage over UFH}

For cost reasons, managed care organizations often recommend LMWH, which can be administered subcutaneously in outpatient settings, over IV UFH administered in the hospital. A retrospective analysis of medical costs from the 1990s in a managed care organization found that bridge therapy with LMWH prior to elective surgery cost an average of $\$ 13,114$ less per patient (in total cost of care) than did bridge therapy with UFH. ${ }^{18}$

\section{LMWH safety issues in valve patients are a myth}

Clinical outcomes were not statistically significantly different for patients receiving LMWH or UFH in the above study. ${ }^{18}$ Nevertheless, there is a widely held notion that LMWH is not safe to use as bridge therapy for patients with mechanical heart valves. Recent prospective bridge 
TABLE 3

Benefits and risks of bridge anticoagulation therapy in prospective clinical trials

\begin{tabular}{|c|c|c|c|c|c|}
\hline Study & $\begin{array}{l}\text { Patients } \\
\text { (N) }\end{array}$ & $\begin{array}{l}\text { Follow-up } \\
\text { (mo) }\end{array}$ & $\begin{array}{c}\text { Reason for } \\
\text { anticoagulation }\end{array}$ & $\begin{array}{c}\text { Thromboembolism } \\
(\%)\end{array}$ & $\begin{array}{c}\text { Major bleeding } \\
(\%)\end{array}$ \\
\hline Douketis et al, $2004^{9}$ & 650 & 0.5 & AF, MHV & $0.6 \%$ & $1.0 \%$ \\
\hline Kovacs et al, $2004^{10}$ & 224 & 3 & $\mathrm{AF}, \mathrm{MHV}$ & $1.3 \%$ & $6.9 \%$ \\
\hline Dunn et al, $2007^{11}$ & 260 & 1 & AF, DVT & $2.3 \%$ & $3.5 \%$ \\
\hline Spyropoulos et al, 2006 & 901 & 1 & AF, MHV, VTE & $1.5 \%$ & $3.3 \%$ \\
\hline Turpie and Douketis, $2004^{13}$ & 220 & 3 & MHV & $0.5 \%$ & $3.5 \%$ \\
\hline Jaffer et al, $2005^{14}$ & 493 & 1 & VTE, CVA, AF, MHV & $0.8 \%$ & $3.2 \%$ \\
\hline
\end{tabular}

$\mathrm{AF}=$ atrial fibrillation; $\mathrm{MHV}=$ mechanical heart valve; DVT = deep vein thrombosis; $\mathrm{VTE}=$ venous thromboembolism; $\mathrm{CVA}=$ cerebrovascular accident $(\mathrm{stroke})$

studies do not support that view, demonstrating that LMWH used as bridge therapy is associated with low risks for thromboembolism and major bleeding even in patients with mechanical valves, ${ }^{9,10,12-14}$ In contrast, recent data on the use of IV UFH for bridging is minimal, with most bridge studies dating to the 1970s. Accordingly, the latest ACCP guidelines for perioperative management of patients on antithrombotic therapy recommend therapeutic-dose LMWH over IV UFH for bridge therapy, including in patients with mechanical heart valves. ${ }^{1}$ Likewise, 2006 guidelines from the American College of Cardiology and American Heart Association on management of patients with valvular heart disease endorse LMWH as an option for bridge therapy. ${ }^{19}$

\section{A PRACTICAL APPROACH TO BRIDGE THERAPY}

A bridge therapy protocol for patients receiving warfarin has been successfully used at the Cleveland Clinic, where I previously practiced. Essentials of the protocol ${ }^{20}$ are summarized here, followed by commentary that draws on additional sources.

\section{Before surgery}

- Discontinue warfarin 5 days before surgery (ie, hold four doses) if the preoperative international normalized ratio (INR) is 2 to 3 , and 6 days before surgery (hold five doses) if the INR is 3 to 4.5 .

- For bridge therapy, start LMWH (enoxaparin 1 $\mathrm{mg} / \mathrm{kg}$ or dalteparin $100 \mathrm{IU} / \mathrm{kg}$ subcutaneously every 12 hours) beginning 36 hours after the last dose of warfarin.

- Give the last dose of LMWH approximately 24 hours prior to surgery.

\section{After surgery}

- For minor surgery, reinitiate LMWH at full dose approximately 24 hours after surgery. For major surgery and for patients at high risk of bleeding, consider using prophylactic doses on the first two postoperative days.
- Discuss the timing of anticoagulant reinitiation with the surgeon.

- Restart warfarin at preoperative dose 1 day after surgery.

- Order daily prothrombin time/INR tests until the patient is discharged and periodically after discharge until the INR is within the therapeutic range.

- Order a complete blood cell count with platelets on days 3 and 7.

- Discontinue LMWH when the INR is between 2 and 3 for 2 consecutive days.

Additionally, the plan should be discussed in advance with the patient, surgeon, and anesthesiologist, along with the risks and benefits associated with LMWH. The patient should receive written instructions for selfadministration and information about signs and symptoms of bleeding and thromboembolism.

\section{When to stop warfarin}

Warfarin should be discontinued far enough in advance of surgery to achieve a preoperative target INR of less than 1.2. ${ }^{21}$ Patients with an initial INR of 2 to 3 tend to achieve that target after discontinuation of warfarin for about 5 days (four doses). A longer wait (6 days, or five doses) is necessary for patients with an initial INR of 3 to 4 . Age is associated with a slower rate of decrease in the INR, and there is wide interpatient variation. The INR should always be checked prior to surgery. ${ }^{21}$

\section{Warfarin need not be stopped for all procedures}

It is commonly assumed that warfarin should be discontinued for any procedure, including minor surgery. But several procedures, listed in Table 4, can be performed safely without discontinuing long-term anticoagulation, as suggested by several literature reviews and comparative studies. ${ }^{22-25}$ Additionally, a 2003 systematic review concluded that major bleeding with continuation of therapeutic oral anticoagulation was rare for patients 
TABLE 4

Procedures that can be performed without discontinuing warfarin ${ }^{22-25}$

\begin{tabular}{llll}
\hline Ophthalmic & Dental & Dermatologic & \\
Cataract surgery & Restorations & Gastrointestinal & \\
Trabeculectomy & Uncomplicated extractions & Mohs' surgery & Diagnostic esophagogastroduodenoscopy \\
& Endodontics & & Colonoscopy without biopsy \\
& Prosthetics & Diagnostic endoscopic retrograde cholangiopancreatography \\
& Periodontal therapy & Biliary stent without sphincterotomy \\
& Dental hygiene & Endoscopic ultrasonography without biopsy \\
& & Push enteroscopy
\end{tabular}

undergoing dental procedures, arthrocentesis, cataract surgery, upper endoscopy, or colonoscopy. ${ }^{26}$

\section{If warfarin is stopped for minor procedures, bridging may be counterproductive}

At the same time, a recent prospective observational study evaluated the effects of brief ( $\leq 5$ days) interruption of warfarin among more than 1,000 patients undergoing minor outpatient procedures and found low rates of both thromboembolism $(0.7 \%)$ and major bleeding $(0.6 \%) .27$ The risk of major bleeding was significantly higher among the small proportion of patients who received bridge therapy with UFH or LMWH. The study concluded that interrupting warfarin for 5 days or less for minor outpatient procedures carries a low risk of thromboembolism and that the risk of clinically significant bleeding should be weighed before bridge therapy is considered in this setting.

\section{When to stop bridge therapy preoperatively}

Bridge therapy with LMWH is commonly discontinued 12 hours before surgery, but it is preferable to discontinue 24 hours before surgery. In a study of preoperative anticoagulant activity in 80 patients, LMWH (enoxaparin $1 \mathrm{mg} / \mathrm{kg}$ ) was administered twice daily and discontinued the night before surgery. ${ }^{28}$ Blood anti-factor Xa levels were measured shortly before surgery, at which time $68 \%$ of patients still had therapeutic levels of anti-Xa. This suggests that discontinuing LMWH too close to the time of surgery can increase the risk of bleeding.

Consistent with these findings, consensus guidelines from the American Society of Regional Anesthesia and Pain Medicine (ASRA) recommend that needle placement for regional anesthesia take place 12 hours after the last dose of LMWH if prophylactic dosing is used and 24 hours after the last dose of LMWH if therapeutic dosing is used (ie, $\geq 1 \mathrm{mg} / \mathrm{kg}$ of enoxaparin every 12 hours). ${ }^{29}$

\section{Dosing and timing of postoperative bridge therapy} Postoperative use of full-dose bridge therapy is associated with increased risks of bleeding, according to a multicenter study of approximately 500 patients who received various doses of UFH or LMWH for bridge therapy. ${ }^{14}$ Patients who received full-dose LMWH or UFH after surgery had a fivefold to sixfold increase in the incidence of major bleeding compared with patients who received prophylactic doses. The study centers that frequently used full-dose bridge protocols were four times as likely to report major bleeding events. In light of these findings, waiting a couple of days after surgery to initiate full-dose bridge therapy is recommended, and prophylactic dosing may be considered in the interim.

The ASRA consensus guidelines recommend that indwelling catheters be removed prior to postoperative reinitiation of twice-daily dosing of LMWH. The first dose of LMWH should be given no sooner than 2 hours after catheter removal. Once-daily dosing of LMWH (European dosing) is acceptable under the ASRA guidelines, but the first dose should be given 6 to 8 hours after surgery and the second dose no sooner than 24 hours later. The guidelines state that once-daily (but not twice-daily) LMWH dosing is acceptable in patients with indwelling catheters; neurological status should be monitored in these patients, and the catheter should be removed 12 to 24 hours after the last dose of LMWH. ${ }^{29}$

\section{PERIOPERATIVE MANAGEMENT OF ANTIPLATELET THERAPY: TYPE OF AGENT MATTERS}

Unlike the considerations with warfarin, the timing of preoperative discontinuation of antiplatelet therapy in patients undergoing noncardiac surgery depends on the type of agent used and its pharmacokinetic actions. Commonly used antiplatelet drugs include aspirin, the thienopyridine agent clopidogrel, and nonsteroidal anti-inflammatory drugs (NSAIDs).

Aspirin works by irreversibly inhibiting platelet cyclooxygenase. The circulating platelet pool is replaced every 7 to 10 days, so aspirin therapy should be discontinued 7 to 10 days before surgery. ${ }^{1}$

NSAIDs reversibly inhibit platelet cyclooxygenase. 
TABLE 5

Preoperative evaluation of patients with stents:A checklist

Determine type of stent(s): Bare metal or drug-eluting? If drug-eluting, sirolimus or paclitaxel?

Determine how long ago each stent was implanted

Determine location of each stent in the coronary circulation

How complicated was the revascularization? Were there any complications (eg, malapposition)?

Is there a prior history of stent thrombosis?

What antiplatelet regimen is being used?

Determine patient's comorbidities to further ascertain risk level (ejection fraction, diabetes, renal insufficiency)

What is the recommended duration of dual antiplatelet therapy for the specific patient at hand?

Consult with patient's cardiologist to review current antiplatelet management and discuss optimal management strategy

Knowing whether a patient is using short- or longacting NSAIDs is important for determining when to discontinue therapy. For a short-acting NSAID such as ibuprofen, discontinuation 24 hours before surgery may be adequate to normalize platelet function. ${ }^{1,30}$

Thienopyridines inhibit adenosine diphosphate receptor-mediated platelet activation and aggregation. Short-acting thienopyridines may be discontinued 24 hours before surgery, but long-acting agents such as clopidogrel should be stopped 7 days prior to surgery (including when used with aspirin as dual antiplatelet therapy), ${ }^{1}$ although some outcomes data suggest that 5 days may be sufficient. ${ }^{31}$

All of these agents should be resumed as soon as adequate hemostasis is achieved after surgery. The ACCP guidelines on perioperative management of antithrombotic therapy recommend resumption of aspirin at the usual maintenance dose the day after surgery, but they make no specific recommendations on when to resume other antiplatelet drugs. ${ }^{1}$

\section{ANTIPLATELET THERAPY: SPECIAL CONSIDERATIONS IN PATIENTS WITH STENTS}

Patients who are on antiplatelet therapy because they have a coronary stent merit special consideration due to the high risk of thrombosis if therapy is interrupted. The risk of stent thrombosis is especially elevated in the postoperative period, particularly if surgery follows soon after stent placement.

Optimal preoperative management of patients with coronary artery stents depends on many factors, as out- lined in Table 5. Some patients carry a wallet card that provides some of this crucial information, such as the type of stent and the date and location of its placement, but speaking with the patient's cardiologist is always recommended. This information, determined in conjunction with the cardiologist, should be used to inform the key perioperative considerations in this setting:

- Relative risks and benefits of stopping versus continuing antiplatelet therapy

- Identification of patients at high risk for a perioperative event after cessation of antiplatelet therapy

- Identification of patients at high risk of bleeding.

\section{Bleeding vs stent thrombosis: Consider relative consequences}

The risk of bleeding varies by individual patient. No laboratory tests are available to determine individual bleeding risk, but the risk of perioperative bleeding increases when two or more antiplatelet agents are used, as in dual antiplatelet therapy with aspirin and clopidogrel..$^{31}$

When balancing risks of bleeding versus thrombotic events, the relative consequences of each event again must be considered. Bleeding is rarely life-threatening in comparison with the potential consequences of stent thrombosis. In a prospective observational study of 2,229 patients who received drug-eluting stents, 29 (1.3\%) developed stent thrombosis during 9-month follow-up. ${ }^{32}$ Among these patients, 20 (69\%) had a nonfatal myocardial infarction and 13 (45\%) died. The most significant independent risk factor for stent thrombosis was premature discontinuation of antiplatelet therapy (hazard ratio $=89.78$ [95\% CI, 29.90-260.60]; $P<.001$ ). Other independent risk factors included renal failure, bifurcation lesions, diabetes, and low ejection fraction.

\section{Premature interruption of antiplatelet therapy: Why it matters}

Abrupt discontinuation of antiplatelet therapy can lead to a rebound effect marked by an inflammatory prothrombotic state, increased platelet adhesion and aggregation, and excessive thromboxane $\mathrm{A}_{2}$ activity. Surgery further increases the prothrombotic and inflammatory state, which, combined with incompletely endothelialized drug-eluting stents, can lead to stent thrombosis and, consequently, myocardial infarction and/or death. ${ }^{33}$

\section{Timing of surgery after stenting: Getting it right}

The US Food and Drug Administration recommends that dual antiplatelet therapy be continued for at least 3 months after placement of a sirolimus-eluting stent and at least 6 months after placement of a paclitaxel-eluting stent. Recent data suggest, however, that this duration of antiplatelet therapy may not be sufficient and that at least 1 year of therapy may be needed. ${ }^{34}$

A recent joint science advisory from the American 
College of Cardiology (ACC) and the American Heart Association (AHA) emphasizes the importance of educating providers about the "potentially catastrophic" risks of premature stopping of thienopyridine therapy in patients with coronary stents. ${ }^{34}$ In addition to recommendations in this joint advisory, the ACC and AHA issued updated guidelines in 2007 on perioperative cardiovascular evaluation and care for noncardiac surgery. ${ }^{35}$ Below is a summary of recommendations on the timing of surgery following stenting in light of these and other sources:

- Following placement of a bare metal stent, elective and nonurgent procedures should be delayed for at least 1 month, according to the ACC/AHA joint advisory, ${ }^{34}$ or at least 6 weeks, according to the ACC/AHA guidelines. ${ }^{35}$ Newer data suggest that the optimal interval for delay is likely to be 3 months. ${ }^{36,37}$

- For patients with recent ( $<6$ weeks) bare metal stent placement who require urgent surgery, dual antiplatelet therapy should be continued during the perioperative period. ${ }^{1}$

- Following placement of a drug-eluting stent, elective and nonurgent procedures should be delayed for at least 12 months. ${ }^{34,35}$

- For patients with recent drug-eluting stent placement in whom surgery cannot be delayed, dual antiplatelet therapy should be continued without interruption if the stent was placed within the prior 6 months. ${ }^{1,35}$ If the stent was placed more than 6 months before urgent surgery, aspirin should be continued without interruption (at $\geq 81 \mathrm{mg} /$ day) and clopidogrel should be continued until 5 days before surgery and resumed as soon as possible after surgery (at a loading dose of $300 \mathrm{mg}$ followed by $75 \mathrm{mg} /$ day). If the surgeon is comfortable continuing dual antiplatelet therapy in a patient whose stent was placed 6 to 12 months earlier, that course should be considered. ${ }^{1}$

It is important to note that the ACC/AHA joint advisory ${ }^{34}$ and other documents have medicolegal implications, so delaying nonurgent surgery for the periods recommended is the most prudent approach.

\section{CONCLUSIONS}

Perioperative management of anticoagulant and antiplatelet therapy is complicated by the paucity of randomized clinical trial data and the risk for serious adverse events. The underlying indications for anticoagulant and antiplatelet therapy vary widely, so the best approach to perioperative management is to involve all members of the health care team-hospitalist, surgeon, cardiologist, and anesthesiologist, together with the patient- to ensure that care is individualized and all relevant considerations are accounted for. Patient and surgical risks can be identified and quantified to some extent, but patients often have greater concerns about the risk of stroke than the risk of bleeding. Ideally, nonemergency surgeries should be scheduled to allow enough time to thoroughly plan the management protocol, reducing risks for bleeding and thrombotic events as much as possible.

\section{DISCUSSION}

Question from the audience: If a patient's INR is 1.3 or 1.4 , rather than the recommended 1.2 , is it necessary to cancel a planned epidural?

Dr. Jaffer: It depends on how comfortable the surgeon or anesthesiologist is with the INR level. Generally, an INR less than 1.5 is probably acceptable, but it depends on the procedure. For a craniotomy, for example, 1.2 is recommended.

Question from the audience: Is it necessary to use anti-Xa levels to guide bridge therapy when administering LMWH or UFH in a patient with a mechanical heart valve?

Dr. Jaffer: It's not generally necessary, except for pregnant women. For most patients, doses are calculated as milligrams of LMWH per kilogram body weight or as International Units of LMWH per kilogram.

Question from the audience: You mentioned medicolegal disputes arising from adverse events associated with bridge therapy, drug discontinuation, or related issues. Who has final responsibility for making decisions about discontinuation of antiplatelet therapy, for example?

Dr. Jaffer: I don't know if it ultimately comes down to just one person. Several physicians should be involved in the decision, and communication protocols within an institution should be very clear. It's important to make certain everyone involved in the decision is reviewing the same literature. The final decision has to be something everyone involved can accept and support.

\section{DISCLOSURES}

Dr. Jaffer has indicated that he has relevant financial relationships with the following commercial interests: grant/research support from Sanofi-Aventis and AstraZeneca; consultant to Sanofi-Aventis, AstraZeneca, and Boehringer Ingelheim; speakers' bureau of Sanofi-Aventis; and board member of the Society for Perioperative Assessment and Quality Improvement (SPAQI) and the Anticoagulation Forum. All conflicts of interest have been resolved.

This article was developed from an audio transcript of Dr. Jaffer's lecture at the 4th Annual Perioperative Medicine Summit. The transcript was edited by the Cleveland Clinic Journal of Medicine staff for clarity and conciseness, and was then reviewed, revised, and approved by Dr. Jaffer.

\section{REFERENCES}

1. Douketis JD, Berger PB, Dunn AS, et al. The perioperative management of antithrombotic therapy: American College of Chest Physicians Evidence-Based Clinical Practice Guidelines (8th Edition). Chest 2008; 133(suppl 6):299S-339S.

2. du Breuil AL, Umland EM. Outpatient management of anticoagulation therapy. Am Fam Physician 2007; 75:1031-1042.

3. Cannegieter SC, Rosendaal FR, Briët E. Thromboembolic and bleeding complications in patients with mechanical heart valve prostheses. Circulation 1994; 89:635-641. 
4. Snow V, Weiss KB, LeFevre M, et al. Management of newly detected atrial fibrillation: a clinical practice guideline from the American Academy of Family Physicians and the American College of Physicians. Ann Intern Med 2003; 139:1009-1017.

5. Genewein U, Haeberli A, Straub PW, Beer JH. Rebound after cessation of oral anticoagulant therapy: the biochemical evidence. Br J Haematol 1996; 92:479-485.

6. Grip L, Blombäck M, Schulman S. Hypercoagulable state and thromboembolism following warfarin withdrawal in post-myocardial-infarction patients. Eur Heart J 1991; 12:1225-1233.

7. Palareti G, Legnani C, Guazzaloca G, et al. Activation of blood coagulation after abrupt or stepwise withdrawal of oral anticoagulants: a prospective study. Thromb Haemost 1994; 72:222-226.

8. Kearon C, Hirsh J. Management of anticoagulation before and after elective surgery. N Engl J Med 1997; 336:1506-1511.

9. Douketis JD, Johnson JA, Turpie AG. Low-molecular-weight heparin as bridging anticoagulation during interruption of warfarin: assessment of a standardized periprocedural anticoagulation regimen. Arch Intern Med 2004; 164:1319-1326.

10. Kovacs MJ, Kearon C, Rodger M, et al. Single-arm study of bridging therapy with low-molecular-weight heparin for patients at risk of arterial embolism who require temporary interruption of warfarin. Circulation 2004; 110:1658-1663.

11. Dunn AS, Spyropoulos AC, Turpie AG. Bridging therapy in patients on long-term oral anticoagulants who require surgery: the Prospective Peri-operative Enoxaparin Cohort Trial (PROSPECT). J Thromb Haemost 2007; 5:2211-2218.

12. Spyropoulos AC, Turpie AG, Dunn AS, et al. Clinical outcomes with unfractionated heparin or low-molecular-weight heparin as bridging therapy in patients on long-term oral anticoagulants: the REGIMEN registry. J Thromb Haemost 2006; 4:1246-1252.

13. Turpie AG, Douketis JD. Enoxaparin is effective and safe as bridging anticoagulation in patients with a mechanical prosthetic heart valve who require temporary interruption of warfarin because of surgery or an invasive procedure. Blood 2004:202s. Abstract 703.

14. Jaffer AK, Brotman DJ, White RH. The perioperative and periprocedural management of warfarin: the PPMW study. J Thromb Haemost 2005; 3(suppl 1). Abstract OR309.

15. Linkins LA, Choi PT, Douketis JD. Clinical impact of bleeding in patients taking oral anticoagulant therapy for venous thromboembolism: a meta-analysis. Ann Intern Med 2003; 139:893-900.

16. Schulman S, Rhedin AS, Lindmarker P, et al. A comparison of six weeks with six months of oral anticoagulant therapy after a first episode of venous thromboembolism: duration of Anticoagulation Trial Study Group. N Engl J Med 1995; 332:1661-1665.

17. Atrial Fibrillation Investigators. Risk factors for stroke and efficacy of antithrombotic therapy in atrial fibrillation: analysis of pooled data from five randomized controlled trials. Arch Intern Med 1994; 154:1449-1457.

18. Spyropoulos AC, Frost FJ, Hurley JS, Roberts M. Costs and clinical outcomes associated with low-molecular-weight heparin vs unfractionated heparin for perioperative bridging in patients receiving long-term oral anticoagulant therapy. Chest 2004; 125:1642-1650.

19. American College of Cardiology/American Heart Association Task Force on Practice Guidelines. ACC/AHA 2006 guidelines for the management of patients with valvular heart disease. Circulation 2006; 114:e84-e231.

20. Jaffer AK, Brotman DJ, Chukwumerjie N. When patients on warfarin need surgery. Cleve Clin J Med 2003; 70:973-984.

21. White RH, McKittrick T, Hutchinson R, Twitchell J. Temporary discontinuation of warfarin therapy: changes in the international normalized ratio. Ann Intern Med 1995; 122:40-42.
22. Konstantatos A. Anticoagulation and cataract surgery: a review of the current literature. Anaesth Intensive Care 2001; 29:11-18.

23. Weibert RT. Oral anticoagulant therapy in patients undergoing dental surgery. Clin Pharm 1992; 11:857-864.

24. Billingsley EM, Maloney ME. Intraoperative and postoperative bleeding problems in patients taking warfarin, aspirin, and nonsteroidal antiinflammatory agents: a prospective study. Dermatol Surg 1997; 23:381-383.

25. Kadakia SC, Angueira CE, Ward JA, Moore M. Gastrointestinal endoscopy in patients taking antiplatelet agents and anticoagulants: survey of ASGE members: American Society for Gastrointestinal Endoscopy. Gastrointest Endosc 1996; 44:309-316.

26. Dunn AS, Turpie AG. Perioperative management of patients receiving oral anticoagulants: a systematic review. Arch Intern Med 2003; 163:901-908.

27. Garcia DA, Regan S, Henault LE, et al. Risk of thromboembolism with short-term interruption of warfarin therapy. Arch Intern Med 2008; 168:63-69.

28. O'Donnell MJ, Kearon C, Johnson J, et al. Brief communication: preoperative anticoagulant activity after bridging low-molecularweight heparin for temporary interruption of warfarin. Ann Intern Med 2007; 146:184-187.

29. Horlocker TT, Wedel DJ, Benzon H, et al. Regional anesthesia in the anticoagulated patient: defining the risks (the second ASRA Consensus Conference on Neuraxial Anesthesia and Anticoagulation). Reg Anesth Pain Med 2003; 28:172-197.

30. Goldenberg NA, Jacobson L, Manco-Johnson MJ. Brief communication: duration of platelet dysfunction after a 7-day course of ibuprofen. Ann Intern Med 2005; 142:506-509.

31. Lecompte T, Hardy JF. Antiplatelet agents and perioperative bleeding. Can J Anaesth 2006; 53(suppl 6):S103-S112.

32. Iakovou I, Schmidt T, Bonizzoni E, et al. Incidence, predictors, and outcome of thrombosis after successful implantation of drugeluting stents. JAMA 2005; 293:2126-2130.

33. Newsome LT, Weller RS, Gerancher JC, Kutcher MA, Royster RL. Coronary artery stents: II. Perioperative considerations and management. Anesth Analg 2008; 107:570-590.

34. Grines CL, Bonow RO, Casey DE Jr, et al. Prevention of premature discontinuation of dual antiplatelet therapy in patients with coronary artery stents: a science advisory from the American Heart Association, American College of Cardiology, Society for Cardiovascular Angiography and Interventions, American College of Surgeons, and American Dental Association, with representation from the American College of Physicians. Circulation 2007; 115:813-818.

35. Fleisher LA, Beckman JA, Brown KA, et al. ACC/AHA 2007 guidelines on perioperative cardiovascular evaluation and care for noncardiac surgery: a report of the American College of Cardiology/American Heart Association Task Force on Practice Guidelines [published correction appears in J Am Coll Cardiol 2008; 52:794797]. J Am Coll Cardiol 2007; 50:1707-1732.

36. Brichon PY, Boitet P, Dujon A, et al. Perioperative in-stent thrombosis after lung resection performed within 3 months of coronary stenting. Eur J Cardiothorac Surg 2006; 30:793-796.

37. Nuttall GA, Brown MJ, Stombaugh JW, et al. Time and cardiac risk of surgery after bare-metal stent percutaneous coronary intervention. Anesthesiology 2008; 109:588-595.

Correspondence: Amir K. Jaffer, MD, Chief, Division of Hospital Medicine, University of Miami Miller School of Medicine, P.O. Box 016760, Miami, FL 33101-6760; ajaffer@miami.edu 